\title{
Challenges in creating environments for SOA learning
}

\author{
Nicolás López, Rubby Casallas and Jorge Villalobos \\ University of los Andes \\ \{ni-lopez,rcasalla,jvillalo\}@uniandes.edu.co
}

\begin{abstract}
SOA is now in widespread use by the industry and is a current area of interest for research. However a currently open area of investigation is how to properly introduce SOA in an IT/CS/SwE type of curriculum.

Software engineering students must develop a series of abilities and skills related to SOA for effective professional practice.

Providing environments where students go beyond learning some concepts and specific technologies to truly apprehend the complexity involved in SOA is a mayor challenge. This environment must be articulated in the context of business needs and other software architecture methodologies. Instructors need a new set of skills and abilities to tackle this challenge.

This position paper discusses what such an environment should provide for students and instructors to effectively develop skills that will enable SOA practice in the business world.
\end{abstract}

\section{Introduction}

The SOA paradigm is currently increasing its role in the industry. Investigation in SOA in all its areas is growing in importance and the subject is of high relevance to SE practice. In particular SOA training and education is an area that needs further research.

At the SIA Technology Management Conference 2006, Rosen and Wang [2] identified that education is the central pillar for the enterprise shift towards SOA. An organization needs to identify educational needs and align an education program with the overall SOA initiative.

At the ASU Workshop on Service-Oriented Architecture and Applications [5], the discussion on SOA education in colleges and universities agreed that education has to be aligned with the current industry trend towards SOA; it needs to be flexible and adaptable; the basic knowledge of SOA is not enough for developing decision making skills at a high level; and that new methodologies and strategies for instruction may be necessary.

Currently SOA education is also heavily promoted by vendors of specific SOA tools such as IBM [3], [4].

In a recent ebizQ webinar [1], "The Path to SOA Enlightenment", noted that the subject of SOA education is now a central topic of interest. A survey to the audience indicated some significant results. The area of most interest for training is SOA design. This area was seen as much more relevant than technologies such as component models, BPM and vendor specific tools. The survey also revealed that topic areas of most relevance were organization, policies and governance, SOA design, and standards.

Our position in this paper is that research in education is an important line for the development of SOA. This research must tackle a series of challenges to create learning environments for students and instructors that effectively develop skills necessary for confronting SOA in professional practice. This environment must help develop skills related to business needs and requirements, infrastructure and technologies, and application integration with SOA. A new set of competences is required from instructors to work in this environment.

The next section presents the challenges for students and instructors in SOA learning. Section 3 presents the pedagogical objectives for implementation SOA solutions. Section 4 presents the characteristics of a learning environment that promotes the development of these objectives. Finally, we present some conclusions.

\section{Challenges}

At University of Los Andes we are attempting to include SOA as part of the Software Architecture course and creating a new elective dedicated to SOA. We have found that including SOA as part of an undergraduate program is a challenge for several reasons.

Issues are related to restrictions of undergraduate programs, intricacy of current technologies, 
implementations and standards for SOA, complexity to create business scenarios in SOA, and high exigency of skills required from instructors for proper SOA teaching.

\subsection{Undergraduate programs}

Software Engineering curricula are tightly packed; many subjects and technologies are covered. Standards such as SWEBOK are so extensive that they lag behind in new approaches such as SOA [7]. These conditions make difficult creating room for a comprehensive review of all architectural paradigms in a typical Software Architecture course.

On the other hand, the time dedicated by students to a 3 credit course makes the implementation of SOA architectures difficult (a credit is 1 hour of lecture and 2 hours of additional work). A single semester might be too short to develop business applications and infrastructure and then integrate them in an SOA environment.

\subsection{Technologies, implementations and standards}

Technologies are complex, and implementation oriented. Reference manuals can be very long and full of details. Students have problems filtering all the information and separating concepts from implementation specific details.

Documentation supporting implementations can be hard to find. Even though sometimes examples can be found, a simple Hello World example is not sufficient to understand how technologies are used in the real world.

Most specific implementations are closed and don't allow students to understand how things are happening. A student faced with the challenge of using a new technology such as Web Services has to spend so much time with implementation details that would not be able to grasp how they can be used effectively in an enterprise.

Even if an implementation is open, tailoring this implementation for educational purposes is very difficult. Students usually face these technologies as very complex black boxes. It is difficult to understand how technology accomplishes its roles such as platform independence without having access to concrete implementation details.

Industry lacks widespread standards, so the question of what is "the right" technology that students must use is a tricky one. Standards are becoming increasingly more complex, reference manuals are long and most of the times focus more on details than on concepts.

\subsection{Business scenarios}

Instructors have a tough time creating SOA scenarios and projects because in the real world these are usually very complex; emulating these conditions is challenging for several reasons. Firstly, they usually involve many applications; secondly, a complete comprehension of the business is usually necessary to recognize the advantages of integration with SOA. Thirdly, time restriction imposed by student dedication to a course limit the possible size of projects. Finally, some benefits of SOA such as reusability can only be experienced over a long period of time.

\subsection{Instructors}

Finally, it is desirable that the instructor has a whole new set of skills to overcome these challenges. First of all, he must have knowledge and abilities in SOA practice in the real world. The instructor should have faced the problems of integration of new and legacy applications. He should be an expert in the technologies, but should not be partial to any of them. He should constantly be studying new technologies and standards; and should be able to spot trends in the industry and bring best practices to the classroom.

In conclusion, the role of the instructor is much more active to achieve the requirements of SOA learning.

\section{Pedagogical Objectives of SOA education}

We believe that having the abilities to use the SOA paradigm in practice is more than learning support technologies such as Web Services and SOAP. A practical approach is necessary where students understand the business restrictions that dictate architectural decisions that lead to SOA implementation.

Students must know the role that SOA can play in an organization and what strategies and methodologies are useful to solve business problems using an SOA approach. Students need an understanding of the mechanisms underlying SOA infrastructure rather than just being able to use some specific tools.

As software engineering professionals students must develop skills in three areas to be able to implement SOA solutions in the real world:

1. Ability to explain business conditions that require SOA and what restrictions they impose.

2. Ability to use the underlying infrastructure that supports SOA environments, and choose and justify the selection of one standard, technology or implementation over another. 
3. Ability to integrate real world applications in an SOA environment understanding the complexity involved.

First of all students should be able to identify situations where integration might be achieved using an SOA approach independently of technology. This requires that students must be faced with real world scenarios; however these scenarios should be simple enough from a business perspective since we don't expect students to spend more time understanding the business processes that the integration exercise.

To properly develop these skills we need environments that are tailor made for educational purposes.

\section{Proposed Environment}

Our proposed environment is explained using the three areas in which students must develop skills. All three must be tackled in parallel; establishing relationships between them and organizing specific courses is still a research question.

\subsection{Understanding Business Conditions}

Students need to interact with cases and projects that have specific goals and help develop specific skills. Cases should be small enough for a single class discussion. A single project that creates a complete SOA solution can help put these skills in practice.

Students must have at their disposition simple exercises that focus on specific skills needed to understand the role of SOA in the enterprise. For example, real case scenarios where SOA has and has not worked could be a source for conceptual discussion.

A useful exercise would focus on the identification of business processes and their relationship with concrete process support applications. In this type of exercise students would have to identify which applications are candidate for SOA integration, what services would be necessary from each application and how would these services be orchestrated. Another type of exercise can have as goal the identification of potential risks of SOA migration.

On the other hand, education requires students to work on projects with simplified SOA situations. In these projects the functionality of applications should be fairly simple; the integration should pose some challenges related to SOA design, and integration requirements for applications.

A good alternative is to use as project a business scenario that is of relative intuitive knowledge to the students. For example, we have proposed as project the implementation of a Web Based e-commerce scenario consisted of product providers, package couriers and on-line intermediaries. The functional requirements are quite simple, users can search for products through the intermediaries and orders have to be dispatched to product providers, finally package couriers must receive orders for dispatching. A simple payment application is used on several points of the process to charge for the services provided by each application to the users.

In SOA scenarios such as this, many applications must interact, and in the real world they would be developed by different groups. Since there are time restrictions and to simulate better real conditions, students are divided so that some groups develop the product providers, while others develop the package couriers. The on-line intermediary is developed partially by Teacher Assistants and is used in the class laboratories.

On a later phase of the project the class discusses and decides on which formats, standards and technologies to use for the integration. Finally each group is in charge of creating the SOA environment independently of which implementations are used.

\subsection{Using Infrastructure}

We want an environment where students can easily use SOA infrastructure and understand what it does for him. This infrastructure should be a laboratory where students can experiment, visualize and manipulate directly simplifications of the elements involved in an SOA infrastructure.

The use of this laboratory must cover all areas of SOA infrastructure, from service call, discovery and location up to orchestration with process execution languages.

The laboratory should be based on reduced versions of industry standards that focus on the most critical concepts and reduce complexity. The standards must have the minimal set of elements and simple composition rules to include the basic concepts behind SOA. These standards may not be as complete as industrial standards, but are straightforward to learn and use. The implementations of these standards must be open to easy modification.

We need to provide tools and frameworks similar to those used by SOA industry based on these simplified standards. The tools and frameworks must be easy to install and should focus on specific cases, instead of trying to provide all possible functionalities.

On the other hand, this laboratory needs to provide visual and easy to use monitoring and management tools so students can actually witness the execution of an SOA scenario. 
Additionally, fully functional and easy to implement tests are necessary for students to set up a scenario and test it. These tests must go beyond proving functional aspects; the tests must evaluate the impact on other quality attributes.

In such an environment students can rapidly create extensions, set up example scenarios, and start playing with the configurations and modifications to the framework that might have an impact on the quality attributes of the scenario. Thus they could easily draw conclusions on the impact that infrastructure can have on SOA solutions.

At University of los Andes we are testing a framework that fulfills most of the requirements of an appropriate laboratory for SOA learning. Tongo [6], is a platform that integrates, summarizes and simplifies technologies and architectures related to Web Services; its main objective is to serve as an infrastructure for student laboratories in SOA. It is based on a flexible and decoupled architecture that eases its evolution.

Tongo has been used by graduate students for the last two semesters; groups of students have created extensions and SOA scenarios in less than a weeks work for a 4 credit course. Its architecture is a lightweight version of commercial Web Services infrastructure based on plug-ins that can be easily explained to students in 15 minutes.

Tongo defines a simplified version of various standards such as TongoSOAP, TongoUDDI, TongoBPEL. The simplifications aim at reducing the complexity of the standards while still maintaining the relevant concepts. For example, TongoSOAP can only handle string messages.

The platform provides a visual console that monitors the execution of the engine and enables tracking and administration of the behavior of the server and all connected components.

We are attempting to bring Tongo to an undergraduate level; a robust implementation is currently in development.

\subsection{Integrating Real World Applications}

This is probably the most difficult challenge of teaching/learning SOA, since in the real world it is legacy applications are a candidate for SOA integration. Replicating these scenarios in a classroom is very difficult because most of the times legacy applications are functionally complex and are implemented in technologies unknown to most students. The time dedicated by students to a course is not sufficient to learn how to use a legacy technology.

The instructor must have experience with SOA in the real world to create an environment where applications are simple enough for students to comprehend the tradeoffs of SOA implementation.

We propose an alternative to working with legacy applications that still preserves some of the characteristics of their integration with SOA. The instructor must provide applications that have a closed implementation and only provide facades for their access. Students must then work in extending these pseudo-legacy applications without modifications to the original source code.

The extensions must focus on aspects such as data transformation, concurrency of service calls, or availability of the application. These pseudo-legacy applications can expose some simple requirements using low level mechanisms, such as streams, with some convention that poses a challenge to students. An exercise can focus on extensions to transform the platform specific calls to services exposed by the application, and transform service responses to specific calls to the application API.

\section{Conclusions}

Education in SOA is an important area of research that still has much work pending. However, there are a lot of challenges to achieve its proper introduction in an undergraduate curriculum.

Environments for education of SOA and its associated technologies have to focus on the development of skills that will be of use to students in their professional practice. We identified three main areas where students need to develop skills related to SOA. These environments need tools, real world cases, scenarios and a whole set of pedagogical strategies to help develop these skills.

Instructors should prepare heavily for the introduction of these new technologies to the classroom. A possible way for instructors to prepare correctly for is to constantly work in projects that with industry partners that include SOA implementations.

The role of the instructor as a mere lecturer needs to change; he needs to be more active in bringing his knowledge from the real world and must be able to keep up with the pace of new advances. This is not only true for SOA but also for other software architecture paradigms that are increasing their role in the industry.

\section{References}

[1] S. Kemsley, "SOA education", ebizQ, The Insider's Guide to Business Integration. April 25, 2006. http://www.ebizq.net/blogs/column2/archives/2006/04/soa e ducation.php 
[2] A. Rosen, Y. Wang, "SOA Education Workshop - PDF", Technology Management Conference, www.sia.com/tmc2006/pdf/SOAEdWorkshop.pdf

[3] M. Stansberry. "Big Blue pushes SOA education" http://search400.techtarget.com/originalContent/0,289142,sid 3 gci1146938,00.html 21 Nov 2005

[4] C. Frye. "Big Blue on mission to dominate SOA space", SearchWebServices.com, 25 May 2005. Available at http://searchdomino.techtarget.com/originalContent/0,28914

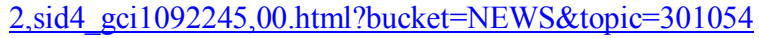

[5] D. Miron. "SOA Education in Colleges and Universities", ASU Workshop on Service-Oriented Architecture and Applications. May 2006, Tempe, Arizona. Available online at

http://asusrl.eas.asu.edu/srlab/activities/SOAWorkshop/ASU Workshop06Proc/13Summary.pdf

[6] Tongo Project Page. University of Los Andes, Bogotá, Colombia. http://agamenon/ tongo/

[7] H. van Vliet, "Some Myths of Software Engineering Education”. ICSE'05, May 15-21, 2005, St. Louis, Missouri, USA. 